\title{
Multilevel Gender-Equitable Norms and Risk of HIV and Herpes Simplex Virus Type 2 Acquisition Among Young South African Women: A Longitudinal Analysis of the HIV Prevention Trials Network 068 Cohort
}

\author{
Paul Wesson, Ph.D. ${ }^{\text {a,* }}$, Sheri A. Lippman, Ph.D. ${ }^{\text {a,b }}$, Torsten B. Neilands, Ph.D. ${ }^{a}$, Rhian Twine, M.P.H. ${ }^{b}$, \\ Jennifer Ahern, Ph.D. ${ }^{\text {c }}$, F. Xavier Gómez-Olivé, Ph.D. ${ }^{\mathrm{b}}$, Dean Peacock, M.S.W. ${ }^{\mathrm{d}, \mathrm{e}}$, \\ Catherine MacPhail, Ph.D. ${ }^{\mathrm{b}}$, Kathleen Kahn, Ph.D., M.P.H., M.B.B.Ch. ${ }^{\text {, }}$, and Audrey Pettifor, Ph.D. ${ }^{\text {b,f }}$ \\ ${ }^{a}$ Center for AIDS Prevention Studies, University of California, San Francisco, San Francisco, California \\ ${ }^{\mathrm{b}}$ MRC/Wits Rural Public Health and Health Transitions Research Unit (Agincourt), School of Public Health, Faculty of Health Sciences, University of the Witwatersrand, \\ Johannesburg, South Africa \\ ${ }^{\mathrm{c}}$ Division of Epidemiology and Biostatistics, School of Public Health, University of California, Berkeley, Berkeley, California \\ ' Sonke Gender Justice, Cape Town, South Africa \\ e School of Public Health, University of Cape Town, Cape Town, South Africa \\ ${ }^{\mathrm{f}}$ Gillings School of Global Public Health, University of North Carolina, Chapel Hill, North Carolina
}

Article history: Received February 6, 2019; Accepted July 11, 2019

Keywords: Adolescent girls; HIV/AIDS; Gender norms; Item response theory; Prevention; Social determinants

\section{A B S T R A C T}

Purpose: Adolescent girls and young women (AGYW) in South Africa experience a disproportionately high burden of HIV acquisition. National HIV prevalence among AGYW increases nearly three-fold during the transition from late teenage years to their early twenties. We investigated whether beliefs about gender equity influence subsequent HIV acquisition among AGYW in South Africa.

Methods: We used data from the HIV Prevention Trials Network 068, a longitudinal conditional cash transfer study of AGYW in Mpumalanga Province, South Africa. Gender-equitable beliefs were measured at the level of the individual and summarized among school peers and adults in the community using the Gender Equitable Men's Scale (GEMS). Generalized estimating equation regression was used to assess the association between individual, peer and community GEMS and HIV incidence, herpes simplex virus type 2 (HSV-2) incidence, and other HIV risk factors while accounting for repeated observations and clustering.

Results: A total of 2,533 AGYW were followed up for up to 5 years. Adjusting for potential confounders, a unit increase in peer GEMS scores (i.e. more equitable) were significantly protective against subsequent HIV acquisition (risk difference $=-.019$; 95\% confidence interval: -.032 , -.006 ) and subsequent HSV-2 acquisition (risk difference $=-.020 ; 95 \%$ confidence interval: $-.040,-.000$ ). Low individual and community GEMS scores were associated with multiple HIV risk factors but not with HIV or HSV-2 incidence directly.

\section{IMPLICATIONS AND CONTRIBUTION}

This study demonstrates that greater endorsement of gender equity in one's peer group is significantly associated with a reduced risk for HIV and herpes simplex virus type 2 acquisition. The results highlight gender norms at multiple levels of influence as a modifiable characteristic that can confer a multitude of benefits to adolescent sexual health.

Conflicts of interest: The authors have no conflicts of interest to disclose.

Disclaimer: The contents are solely the responsibility of the authors and do not necessarily represent the views of the funding institutions.

* Address correspondence to: Paul Wesson, Ph.D., Center for AIDS Prevention Studies/Prevention Research Center, University of California, San Francisco, 550 16th St., 3rd Floor, San Francisco, CA 94158.

E-mail address: paul.wesson@ucsf.edu (P. Wesson). 
Conclusion: School-level peer endorsement of gender equity may be protective against HIV and HSV-2 incidence among AGYW. Interventions that increase gender equity at the individual level and at the level of the social environment, particularly among school peers, have the potential for protective effects on the health of AGYW.

South Africa has the largest HIV epidemic in the world, accounting for nearly $20 \%$ of people living with HIV (PLWH) and $15 \%$ of new HIV acquisitions [1]. South African women and girls bear the largest burden of disease, accounting for $23 \%$ of all female PLWH and 12\% of all PLWH, globally [2]. Among youth aged 15-19 years in sub-Saharan Africa, females account for $75 \%$ of new HIV acquisitions [2]. A 2012 National HIV prevalence, incidence, and behavior survey found that the HIV prevalence among adolescent girls aged 15-19 years in South Africa was $5.6 \%$ and more than tripled to $17.4 \%$ for young women aged 20-24 years [3]. This same national survey found that in this age range, 15-24 years, HIV incidence was over four times higher in young women compared with young men. These statistics underscore the need to identify modifiable risk factors that affect female vulnerability to HIV acquisitions, especially during the developmental period encompassing late adolescence and young adulthood [4].

The social environment of adolescent girls and young women (AGYW) may play a key role in vulnerability to HIV acquisitions. Prior research on sexuality among adolescent girls in South Africa points to a strong peer influence, promoting sexual activity and acceptance of intimate partner violence (IPV) [5-7]. More recent research from South Africa de-emphasizes the direct influence of peers on relationship dynamics, in favor of personal agency. Research by Jewkes et al. [8,9] suggest that young women in South Africa demonstrate strong personal agency in courtship and partner selection; however, this agency appears to give way to traditional notions of masculinity (i.e., the man makes the decisions for the woman) when in relationships. This deference to male authority in relationships (even to the point of physical violence) can be tolerated by the individual young woman for the sake of self-esteem, peer-esteem, and socioeconomic vulnerability, as well as by influential adults in her social environment (e.g. parents). The multilevel influence of one's social environment on what behavior is acceptable and tolerated in interpersonal romantic relationships speaks to the broader context of societal gender norms. Gender norms, social norms defining acceptable behavior for men, women, and relationship dynamics, have also been linked to individual behaviors associated with increased risk of HIV acquisition, primarily among adults $[10,11]$. The interpersonal power imbalance that can result from gender norms has been hypothesized to increase the risk of HIV infection among women due, in part, to their diminished ability to advocate for consistent condom use [7,12]. These interpersonal power imbalances may also increase the risk of sexually transmitted infections such as herpes simplex virus type 2 (HSV-2), which in turn increase the risk for HIV infection. No study, to our knowledge, has explored the relationship of community gender norms, gender norms held by peers, and personally held beliefs about gender with risk of HIV acquisition in an AGYW population.

To examine these relationships, we merged data from a conditional cash transfer randomized controlled trial (RCT) (HIV
Prevention Trials Network [HPTN] 068), with data from a separate cluster RCT of community mobilization (CM) to investigate how gender norms contribute to HIV acquisition among AGYW in rural Mpumalanga Province, South Africa. We hypothesized that AGYW participating in HPTN 068 who endorsed genderequitable beliefs would have a lower risk for HIV acquisition and HSV-2 acquisition, compared with AGYW participating in HPTN 068 who did not endorse gender-equitable beliefs. We further hypothesized that exposure to gender-equitable norms in the social environment would be inversely associated with HIV risk by promoting individual protective behaviors. Specifically, we hypothesized that exposure to more gender-equitable norms from (female) school peers would be associated with reduced HIV and HSV-2 acquisition and reduced HIV-risk behaviors. We hypothesized the same association would hold for exposure to more equitable gender norms among adult males and adult females in the community.

\section{Methods}

\section{Study design}

From March 2011 to December 2012, the HIV Prevention Trials Network (HPTN) 068 study enrolled a longitudinal cohort of AGYW in the Agincourt Health and Demographic Surveillance System in rural Bushbuckridge subdistrict in Mpumalanga province, South Africa [13]. The cohort consisted of 2,533 study participants between the ages of 13 and 20 years who were enrolled in high school grades 8-11. There were 90 high schools represented by the HPTN 068 study participants (mean number of HPTN 068 participants per school: 143; interquartile range: 86-151). The RCT aimed to measure the effect of providing cash transfers, conditional on school attendance, on risk of HIV acquisition. HPTN 068 excluded YW who were pregnant or married at baseline, as well as those who did not have a parent/guardian in the household. Full details of this study are described elsewhere [14].

\section{Study participants}

HPTN 068 enrollment visits began in 2011, and follow-up visits occurred for every subsequent year that the AGYW was in high school (up to grade 12). Follow-up visits for the original RCT concluded in 2015. At each visit, a survey was administered to the AGYW via an audio computer-assisted self-interview, and the participants were tested for HIV and HSV-2 infection. HSV-2 testing was performed only at follow-up visits if the previous visit test result was nonreactive. Following the end of the conditional cash transfer intervention, a postintervention study visit occurred which also included biomarker testing and the audio computer-assisted self-interview study questionnaire. 
Simultaneous to the HPTN 068 study, a community RCT was also underway in the Agincourt Health and Demographic Surveillance System. The purpose of the community RCT was to determine whether a CM intervention designed to raise consciousness and community action around the intersection of HIV and gender norms impacted HIV testing uptake in 11 intervention communities compared to 11 comparison communities. Cross-sectional surveys were conducted before $(\mathrm{n}=1,181 ; 600$ men and 581 women) and after ( $\mathrm{n}=1,403 ; 693$ men and 710 women) the 2-year intervention (2012-2014). The number of survey respondents per village ranged from 39 to 55 . Full details of this community RCT are described elsewhere [15,16]. The multiple sources of data and the longitudinal assessment of participants are depicted in Figure 1.

\section{Measurements}

We used the Gender Equitable Men's Scale (GEMS) to measure gender-equitable beliefs in the study population-in both the HPTN surveys and in the community surveys. GEMS is a validated 24-item instrument assessing respondents' endorsement of statements pertaining to gender roles in the household and the community (e.g., "Changing diapers, giving a bath, and feeding kids are the mother's responsibility.") [11,17]. For each item, respondents can respond "Agree a lot (1)", "Somewhat agree (2)", or "Do not agree at all (3)". A higher score, therefore, represented more gender-equitable beliefs. The original 24 -item GEMS instrument was restricted to 13 items based on a previous psychometric analysis indicating improvement in the properties of the scale for females when the remaining 11 items were removed (Figure 2) [16].

Item response models (IRMs) were used to score the GEMS instrument. IRMs assess a person's ability (or proficiency) in a given subject area. IRMs score a person's ability according to which questions (or items) they answer correctly, as opposed to how many are answered correctly [18,19]. Difficult items are given greater weight in calculating a person's IRM score when answered correctly, relative to easier items. Because GEMS items are not inherently "correct" or "incorrect," we coded responses so that nonendorsement of gender inequitable statements (i.e., responding "Do not agree at all" to any of the GEMS items) were given greater weight than endorsement of these gender inequitable statements. A higher IRM GEMS score therefore reflected more gender-equitable norms/attitudes. IRMs indicated no distinguishable difference between the "Agree a lot" and "Somewhat Agree" categories, which were then collapsed into a single "Agree" category. IRMs were estimated in R version 3.4.1 using the Test Analysis Modules ('TAM') package [20,21]. Units in IRMs are logits, defined as the natural log of the odds ratio. The logit scale is standardized for the population and instrument; negative logits indicate relatively easy items (or for a person's score, a person's relative inability to perform on an instrument) and positive logits indicate relatively harder items (or for a person's score, a person's relative ability to perform on an instrument) [22]. Logits calculated for individual respondents can be compared to logits calculated for individual items to estimate the probability that a given respondent would endorse that item. Peer GEMS scores were calculated by averaging the GEMS scores of the other AGYW in each respondent's school who were also HPTN 068 participants, for each time point. The calculation of the peer GEMS scores for a given AGYW did not include her own GEMS score. The GEMS instrument was also administered to community members from the CM study [23]. For each community, by sex, GEMS scores were averaged together so that each community had male and female GEMS scores at both community survey time points. Community GEMS scores were assigned to each AGYW from HPTN 068, based on the community she resided in for each year of the study.

To assess HIV serostatus, two HIV rapid tests were conducted at the study site at all study visits (the Determine HIV-1/2 test [Alere Medical Co, Matsudo-shi, Chiba, Japan] and the Uni-gold Recombigen HIV test [Trinity Biotech, Bra, County Wicklow, Ireland]). When one or both rapid tests were positive, a confirmatory HIV test was done using the GS HIV-1 western blot assay
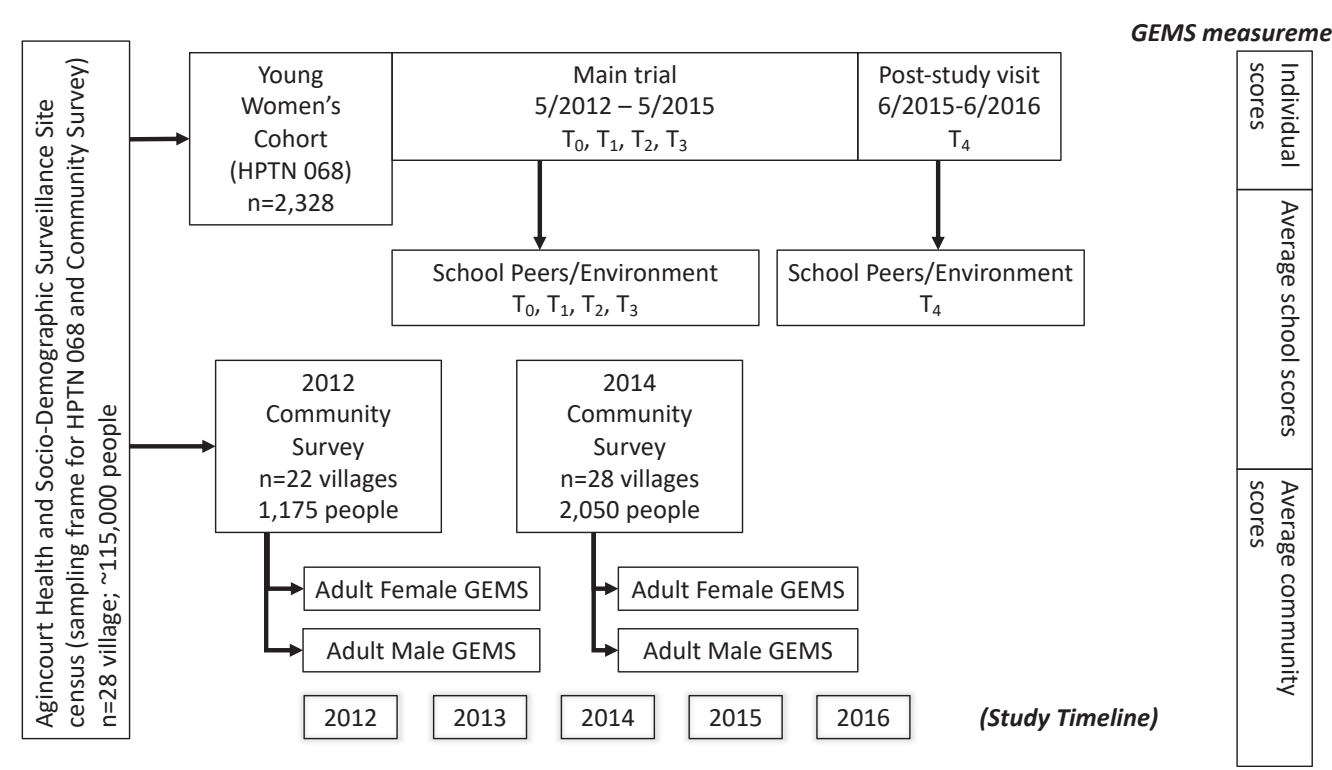

Figure 1. Integration of multiple data sources for longitudinal multilevel analysis of the Gender Equitable Men's Scale. GEMS = Gender Equitable Men's Scale; T0 = baseline study visit (HPTN 068); T1 = second study visit (HPTN 068); T2 = third study visit (HPTN 068); T3 = fourth study visit (HPTN 068); T4 = fifth study visit, postintervention visit (HPTN 068). 


\begin{tabular}{|c|c|c|}
\hline \multicolumn{3}{|c|}{ Gender Equitable Men's Scale items } \\
\hline \multicolumn{3}{|c|}{ 1. It is the man who decides what type of sex to have. } \\
\hline \multicolumn{3}{|c|}{ 2. Men are always ready to have sex. } \\
\hline \multicolumn{3}{|c|}{ 3. Men need sex more than women do. } \\
\hline \multicolumn{3}{|c|}{ 4. A woman should not initiate sex. } \\
\hline \multicolumn{3}{|c|}{ 5. A woman who has sex before she marries does not deserve respect. } \\
\hline \multicolumn{3}{|c|}{ 6. Women who carry condoms on them are easy. } \\
\hline \multicolumn{3}{|c|}{ 7. Only when a woman has a child is she a real woman. } \\
\hline \multicolumn{3}{|c|}{ 8. A real man produces a male child. } \\
\hline \multicolumn{3}{|c|}{ 9. Changing diapers, giving a bath, and feeding kids are the mother's responsibility. } \\
\hline \multicolumn{3}{|c|}{ 10. A woman's role is taking care of her home and family. } \\
\hline \multicolumn{3}{|c|}{ 11. The husband should decide to buy the major household items. } \\
\hline \multicolumn{3}{|c|}{ 12. A man should have the final word about decisions in his home. } \\
\hline \multicolumn{3}{|c|}{ 13. A woman should obey her husband in all things. } \\
\hline \multicolumn{3}{|c|}{ Item Response Theory scores (as logits) } \\
\hline & $\begin{array}{c}\text { Enrollment } \\
\text { Mean } \pm \text { std. dev. } \\
\text { [Interquartile Range] } \\
\mathrm{N}=2,533\end{array}$ & $\begin{array}{c}\text { Final Study Visit } \\
\text { Mean } \pm \text { std. dev. (Range) } \\
\text { [Interquartile Range] } \\
\mathrm{N}=2,185\end{array}$ \\
\hline Individual score & $\begin{array}{l}0.032 \pm 1.104 \\
{[-0.619,0.734]}\end{array}$ & $\begin{array}{l}0.816 \pm 1.349 \\
{[0.046,1.560]}\end{array}$ \\
\hline Peer environment score & $\begin{array}{l}0.032 \pm 0.180 \\
{[-0.058,0.148]}\end{array}$ & $\begin{array}{ll}0.795 & 0.396 \\
{[0.693,} & 0.928]\end{array}$ \\
\hline Female community GEMS & $\begin{array}{l}0.011 \pm 0.264 \\
{[-0.122,0.082]}\end{array}$ & $\begin{array}{c}0.117 \pm 0.160 \\
{[-0.010,0.0221]}\end{array}$ \\
\hline Male community GEMS & $\begin{array}{c}0.057 \pm 0.32 \\
{[-0.142,0.203]}\end{array}$ & $\begin{array}{c}-0.132 \pm 0.229 \\
{[-0.263,-0.031]}\end{array}$ \\
\hline
\end{tabular}

Figure 2. Gender Equitable Men's Scale item and Item Response Theory scores.

(Bio-Rad Laboratories Inc, Redmond, WA). HSV-2 infection was also assessed at required study visits using the Herpes Simplex Virus Type 2 IgG ELISA assay (Kalon Biological Ltd, Guildford, UK) [14].

For this analysis, we used the following variables from the $\mathrm{CM}$ study: CM study arm (intervention or control group), GEMS score for adult males and females separately. All other variables used in this analysis were taken from the HPTN 068 study.

\section{Analysis}

We used generalized estimating equation (GEE) logistic regression, with log link and binomial distribution, to measure the association between gender-equitable beliefs (individual level, peer level, community level) and HIV or HSV-2 incidence. AGYW with prevalent infections at enrollment were dropped from the analytic data set. Based on a test of the intraclass correlation, the school was selected as the unit of clustering. Ensuring temporality, GEMS scores were lagged by one time point; the exposure was GEMS score (individual, peer, or community) from the previous time point and the outcome was current HIV or HSV-2 status. If a participant had missing data on GEMS at one time point, but had a previous GEMS score within one year, we carried forward her measurement to maintain our analytic sample assuming that these scores would not change significantly within a year. Adjusted GEE regression models controlled for reporting ever having sex (at the previous time point), study arm for the conditional cash transfer intervention (intervention or control group), study arm for the CM intervention (intervention or control group), age at baseline, study visit (i.e. time), current grade in school, and if the primary source of income came from formal employment.
The margins command in Stata, version 15, was used to convert risk ratios into risk differences [24]. Risk differences estimate the excess risk of the outcome that is associated with the exposure and provide an assessment of the strength of the relationship that is more relevant to population health/public health [25]. We also examined evidence for two-way interaction between GEMS measured at multiple levels (individual, school peers, community adults).

\section{Ethics approval}

Institutional review board approval for the HPTN 068 cohort study was obtained from both the University of North Carolina at Chapel Hill and the University of the Witwatersrand Human Research Ethics Committee. Institutional review board approval for the community randomized trial was obtained from the University of North Carolina at Chapel Hill, the University of the Witwatersrand Human Research Committee, and the Mpumalanga Department of Health and Social Development Research Committee.

\section{Results}

The HPTN 068 cohort included 2,533 AGYW followed up for up to five years. The average age at enrollment was 15.5 years (range: $13-21$ years) and at the end of the study was 20.2 years (range: 17-26 years) (Table 1). At enrollment, just over one quarter of the sample $(n=683)$ reported having had sex, with the mean age of sexual debut at 14.5 years. The number of AGYW reporting sexual debut increased to 1,246 at the final study visit, roughly half of the study population, with the mean age of sexual 
Table 1

Demographic and behavioral characteristics of young women enrolled in HPTN 068 at enrollment and the final study visit

\begin{tabular}{|c|c|c|c|c|}
\hline \multirow[t]{2}{*}{ Variable } & \multicolumn{2}{|c|}{ Enrollment } & \multicolumn{2}{|c|}{$\begin{array}{l}\text { Final study } \\
\text { visit }\end{array}$} \\
\hline & $N$ & Percent & $n$ & Percent \\
\hline Age $($ mean \pm SD) & 2,533 & $15.5 \pm 1.7$ & 2,533 & $20.2 \pm 1.5$ \\
\hline Age at sexual debut (mean \pm SD) & 683 & $14.5 \pm 3.8$ & 1,246 & $17.2 \pm 2.6$ \\
\hline \multicolumn{5}{|l|}{ Conditional cash transfer } \\
\hline Control & 1,272 & 50.2 & 1,072 & 49.1 \\
\hline Intervention & 1,261 & 49.8 & 1,113 & 50.9 \\
\hline \multicolumn{5}{|l|}{ Community mobilization village } \\
\hline Control village & 1,424 & 56.2 & 1,211 & 55.4 \\
\hline Intervention village & 1,109 & 43.8 & 974 & 44.6 \\
\hline \multicolumn{5}{|l|}{ Education (currently enrolled) } \\
\hline Grade 8 & 640 & 25.3 & 0 & .0 \\
\hline Grade 9 & 682 & 26.9 & 0 & .0 \\
\hline Grade 10 & 699 & 27.6 & 12 & 1.17 \\
\hline Grade 11 & 512 & 20.2 & 153 & 14.9 \\
\hline Grade 12 & 0 & .0 & 358 & 34.8 \\
\hline University & 0 & .0 & 506 & 49.2 \\
\hline \multicolumn{5}{|l|}{ HIV prevalence } \\
\hline Negative & 2,448 & 96.8 & 2,245 & 88.6 \\
\hline Positive & 81 & 3.2 & 288 & 11.4 \\
\hline \multicolumn{5}{|l|}{ HSV-2 prevalence } \\
\hline Negative & 2,409 & 95.3 & 2,195 & 86.7 \\
\hline Positive & 120 & 4.7 & 338 & 13.3 \\
\hline \multicolumn{5}{|l|}{ Any condomless sex in the last } \\
\hline No & 2,309 & 91.9 & 1,518 & 78.6 \\
\hline Yes & 204 & 8.12 & 413 & 21.4 \\
\hline \multicolumn{5}{|l|}{$\begin{array}{l}\text { Experienced physical IPV in the last } \\
12 \text { months }\end{array}$} \\
\hline No & 2,208 & 89.1 & 1,740 & 90.5 \\
\hline Yes & 269 & 10.9 & 182 & 9.5 \\
\hline \multicolumn{5}{|l|}{ Sexual concurrency } \\
\hline No & 2,263 & 89.3 & 1,916 & 87.7 \\
\hline Yes & 270 & 10.7 & 269 & 12.3 \\
\hline \multicolumn{5}{|l|}{$\begin{array}{l}\text { Sexual partner is } \geq 5 \text { years older } \\
\quad \text { (intergenerational sex) }\end{array}$} \\
\hline No & 2,395 & 94.6 & 1,750 & 80.1 \\
\hline Yes & 138 & 5.5 & 435 & 19.9 \\
\hline
\end{tabular}

HSV-2 = herpes simplex virus type 2 ; IPV = intimate partner violence; SD = standard deviation.

debut at 17.2 years. Enrollment in secondary education was an eligibility criterion; $40 \%$ of the full study population was still enrolled in school at the final visit (the postintervention visit), including grade 10 through university.

At the time of enrolment, over $3 \%$ of the study population had already acquired HIV $(n=81)$. By the final study visit, HIV prevalence had increased to $11.4 \%$, with 207 incident cases, resulting in a cumulative incidence of HIV during the study period of $8.5 \%$. In comparison, $4.7 \%$ of the study population had acquired HSV-2 at the time of enrollment $(n=120)$. By the final visit, $13.3 \%$ of participants had acquired HSV-2, with 218 incident cases, resulting in a cumulative incidence of HSV-2 during the study period of $9.5 \%$ (Table 1 ). Reporting of some HIV risk behaviors tended to increase over the study period. Reports of any unprotected sex in the three months preceding the survey increased from $8.1 \%$ to $21.4 \%$; any experiences with physical IPV remained similar at enrollment and the final visit $(10.9 \%$ vs. 9.5\%); concurrent sexual partnerships increased over the study period (10.7\% vs. $12.3 \%$ ); and intergenerational sex (i.e. sexual relationship with a partner that is at least 5 years older) increased over the study period from $5.5 \%$ to $19.9 \%$.

GEMS scores of AGYW increased over follow-up, indicating increasing endorsement of gender equitable statements over time (Figure 2). Bivariate and multivariate analyses indicate individual GEMS scores were not associated with HIV or HSV-2 incidence (Table 2). However, in multivariate models, a logit increase in the peer GEMS scores (demonstrating increasing endorsement of gender equity) was significantly associated with a $2 \%$ reduced risk (adjusted risk difference $[\mathrm{aRD}]=-.019$; $95 \%$ confidence interval $[\mathrm{CI}]:-.032,-.006)$ of HIV acquisition during the five-year period of observation. Higher peer environment GEMS scores (greater endorsement of gender equity) were also significantly associated with a reduced risk for HSV-2 acquisition $(\mathrm{aRD}=-.020 ; 95 \% \mathrm{CI}:-.040,-.000)$ during the five-year period of observation. Community GEMS scores were not associated with HIV or HSV-2 acquisition. A modest interaction effect was observed between individual and peer environment GEMS scores and subsequent HIV acquisition (Figure 3). AGYW at the lowest levels (least equitable) of both individual and peer GEMS scores had the highest predicted probability of HIV acquisition, between $10 \%$ and 15\%. Regardless of the individual GEMS score, the peer environment GEMS score becomes protective at -.11 logits (i.e. approximately equivalent to not endorsing three to four of the thirteen inequitable items), reducing the predicted probability of HIV acquisition to less than $5 \%$ during the five-year period of observation.

Higher individual GEMS scores, peer GEMS scores, and community GEMS scores were associated with a reduced risk for HIV risk behaviors and other potential HIV risk factors. For all adjusted GEE models, higher individual GEMS scores were significantly associated with a reduced risk of unprotected sex $(\mathrm{aRD}=-.007$; $95 \%$ CI: $-.013,-.000)$, IPV (aRD $=-.013 ; 95 \% \mathrm{CI}:-.022,-.005)$, and sexual concurrency ( $\mathrm{aRD}=-.015 ; 95 \% \mathrm{CI}:-.023,-.008$ ). Higher female community GEMS scores were protective against unprotected sex ( $\mathrm{aRD}=-.054 ; 95 \% \mathrm{CI}:-.107,-.001)$, whereas higher male community GEMS scores were protective against sexual concurrency (aRD $=-.050 ; 95 \% \mathrm{CI}:-.094,-.005)$. There was no association between GEMS score (individual, peer, community) and intergenerational sex.

\section{Discussion}

In this study, we investigated how individual, peer, and community gender norms contribute to HIV acquisition among AGYW in rural Mpumalanga Province, South Africa. Our results show that greater endorsement of gender-equitable beliefs among school peers may be protective against individual HIV and HSV-2 acquisition among young women in HPTN 068. A unit increase in the peer GEMS score was associated with a $2 \%$ absolute reduction in risk for HIV acquisition. The cumulative incidence of HIV among HPTN 068 participants was 8.5\%. Therefore, a risk difference of $-2 \%$ indicates that a one unit increase in peer GEMS could reduce the HIV incidence from $8.5 \%$ to $6.5 \%$, corresponding to a significant decrease in new infections in the broader population of young adult females in South Africa. We estimated a similar magnitude of association between peer GEMS scores and HSV-2 incidence, speaking to the broader potential benefit of equitable peer gender norms and sexual health among AGYW.

In contrast to our initial hypothesis, we did not find a direct association between individual-level gender norms and either HIV or HSV-2 acquisition. However, our adjusted models did indicate an interaction effect between individual and peer GEMS scores on HIV risk such that the risk of HIV acquisition is influenced by both the individual GEMS score and the peer GEMS 
Table 2

Bivariate and multivariate associations between Gender Equitable Men's Scores and HIV and HIV-related outcomes, over a five-year period of observation

\begin{tabular}{|c|c|c|c|c|}
\hline Outcome & Main exposure & $\begin{array}{l}\text { Sample size }{ }^{a} \\
\text { AGYW (schools) }\end{array}$ & $\begin{array}{l}\text { Unadjusted risk } \\
\text { difference }(95 \% \mathrm{CI})\end{array}$ & $\begin{array}{l}\text { Adjusted risk } \\
\text { difference }(95 \% \mathrm{CI})\end{array}$ \\
\hline \multirow[t]{4}{*}{ HIV incidence $^{b}$} & Individual GEMS score & $2,364(49)$ & $.001(-.002, .004)$ & $.001(-.003, .004)$ \\
\hline & Peer GEMS score & $2,364(49)$ & $-.002(-.019, .015)$ & $-.019(-.032,-.006)^{* *}$ \\
\hline & Male community GEMS score & $2,194(46)$ & $.001(-.017, .019)$ & $.009(-.008, .027)$ \\
\hline & Female community GEMS score & $2,194(46)$ & $.014(-.011, .039)$ & $-.002(-.031, .027)$ \\
\hline \multirow[t]{4}{*}{ HSV-2 incidence } & Individual GEMS score & $2,288(44)$ & $-.002(-.005, .002)$ & $.000(-.002, .003)$ \\
\hline & Peer GEMS score & $2,288(44)$ & $-.030(-.047,-.013)^{* * *}$ & $-.020(-.040, .000)^{*}$ \\
\hline & Male community ${ }^{\mathrm{c}}$ GEMS score & $2,073(41)$ & $.004(-.011, .018)$ & $.008(-.007, .022)$ \\
\hline & Female community ${ }^{\mathrm{C}}$ GEMS score & $2,073(41)$ & $-.000(-.019, .018)$ & $-.010(-.030, .009)$ \\
\hline \multirow[t]{4}{*}{ Any unprotected sex in last 3 months } & Individual GEMS score & $2,433(50)$ & $-.008(-.016, .001)$ & $-.007(-.013,-.000)^{*}$ \\
\hline & Peer GEMS score & $2,433(50)$ & $.028(-.010, .066)$ & $-.027(-.064, .012)$ \\
\hline & Male community GEMS score & $2,243(48)$ & $-.017(-.079, .046)$ & $.031(-.026, .087)$ \\
\hline & Female community GEMS score & $2,243(48)$ & $-.014(-.112, .085)$ & $-.054(-.107,-.001)^{*}$ \\
\hline \multirow[t]{4}{*}{ Physical IPV in last 12 months } & Individual GEMS score & $2,427(50)$ & $-.020(-.030,-.010)^{* * *}$ & $-.013(-.022,-.005)^{* *}$ \\
\hline & Peer GEMS score & $2,427(50)$ & $-.134(-.186,-.083)^{* * *}$ & $.023(-.031, .077)$ \\
\hline & Male community GEMS score & $2,243(48)$ & $.069(.027, .112)^{* * *}$ & $.023(-.011, .057)$ \\
\hline & Female community GEMS score & $2,243(48)$ & $-.022(-.073,-.028)$ & $-.023(-.074, .028)$ \\
\hline \multirow[t]{4}{*}{ Sexual concurrency ${ }^{\mathrm{d}, \mathrm{e}}$} & Individual GEMS score & $2,487(50)$ & $-.020(-.031,-.010)^{* * *}$ & $-.015(-.023,-.008)^{* * *}$ \\
\hline & Peer GEMS score & $2,487(50)$ & $-.048(-.085,-.011)^{* *}$ & $-.031(-.066,-.003)$ \\
\hline & Male community GEMS score & $2,243(48)$ & $-.015(-.046, .015)$ & $-.050(-.094,-.005)^{*}$ \\
\hline & Female community GEMS score & $2,243(48)$ & $.000(-.037, .037)$ & $.019(-.030, .069)$ \\
\hline \multirow[t]{4}{*}{ Intergenerational sex } & Individual GEMS score & $2,487(50)$ & $.000(-.008, .008)$ & $-.001(-.008, .005)$ \\
\hline & Peer GEMS score & $2,487(50)$ & $.060(.023, .096)^{* * *}$ & $.007(-.032, .045)$ \\
\hline & Male community GEMS score & $2,317(48)$ & $-.017(-.048, .015)$ & $.010(-.026, .046)$ \\
\hline & Female community GEMS score & $2,317(48)$ & $.034(-.009, .078)$ & $-.021(-.065, .023)$ \\
\hline
\end{tabular}

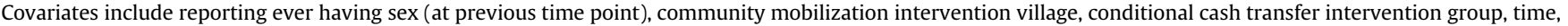
age at baseline, current employment as primary source of income, and current grade in school. Model uses school as the clustering variable.

AGYW = adolescent girls and young women; GEMS = Gender Equitable Men's Scale; HSV-2 = herpes simplex virus type 2; IPV = intimate partner violence).

${ }^{*} p<.05 ;{ }^{* *} p<.01 ;{ }^{* * *} p<.001$.

a Sample sizes are in reference to adjusted models.

b Adjusted model includes interaction between individual GEMS score and peer GEMS score.

c To achieve model convergence, these models assumed an independent correlation structure. All other models assumed an exchangeable correlation structure.

d Models with community GEMS score include an interaction term between Male Community GEMS score and Female Community GEMS score.

e Poisson distribution used in place of log binomial to allow model convergence.

score. We did not observe any association between community GEMS scores and HIV acquisition. This may suggest that while the social environment influences individual outcomes, the more proximal social environment (the peer environment) is most influential at this stage in the life course.

Individual level GEMS scores were protective against behavioral risk factors for HIV acquisition; this is consistent with previous research by Gottert et al. [26], which shows that less endorsement of gender-equitable beliefs was associated with greater odds of sexual concurrency and perpetrating IPV among South African men. Similarly, results from a nationally representative sample of 15- to 24-year-old AGYW in South Africa found that women reporting limited sexual power were not more likely to acquire HIV but were more likely to report inconsistent condom use [12].

In explanation of our results, we hypothesize that individually held beliefs directly influence potential risk factors that are relatively within a person's control (e.g. condomless sex, partner selection, multiple sexual partners). These individually held beliefs may have less influence on biological outcomes (e.g. HIV acquisition) that are both the result of individual behaviors and a complex web of interconnected biological and social risks (e.g. co-occurrence of a sexually transmitted infection, prevalence of HIV in the network of available sexual partners) [27]. Our results suggest that gender-equitable beliefs at different levels of influence impact different behaviors and HIV risk factors. Further decomposition of these different levels of influence could inform and focus intervention efforts that yield the greatest health benefits.

\section{Limitations}

The peer GEMS score was used as a proxy for the peer social environment; however, this variable may only approximate the school environment (among female students) and be less informative for some women depending on the amount of time they are in school or whether their school peers in 068 are also social peers. Notably, HPTN 068 did not include a formal social network analysis; we therefore cannot determine that the peers included in the peer GEMS scores are direct peers/social connections in the AGYW's personal social network. Similarly, the peer GEMS scores only reflect the aggregated scores among other female (HPTN 068) students. Male GEMS scores, among school peers, are absent from this analysis. AGYW may be significantly influenced by the gender-equitable beliefs held by their male counterparts. In fact, South African research has shown a clustering of sexual practices among men in connection to gender norms (including IPV, sexual concurrency, alcohol abuse, and condomless sex) [9], which in turn can place women at increased risk for HIV acquisition.

This study aggregated data from adult members of the communities to approximate community gender norms. Adults participating in the CM study may not be influential in the social environment of the AGYW. AGYW may be more influenced by the gender norms held by specific leaders within the community (e.g. teachers, religious figures, community leaders) than by the gender norms held by the "average" adult community member.

We designed our analysis plan to leverage the strengths of the longitudinal study design. We ensured temporality by dropping prevalent HIV and HSV-2 infections at enrollment from the 


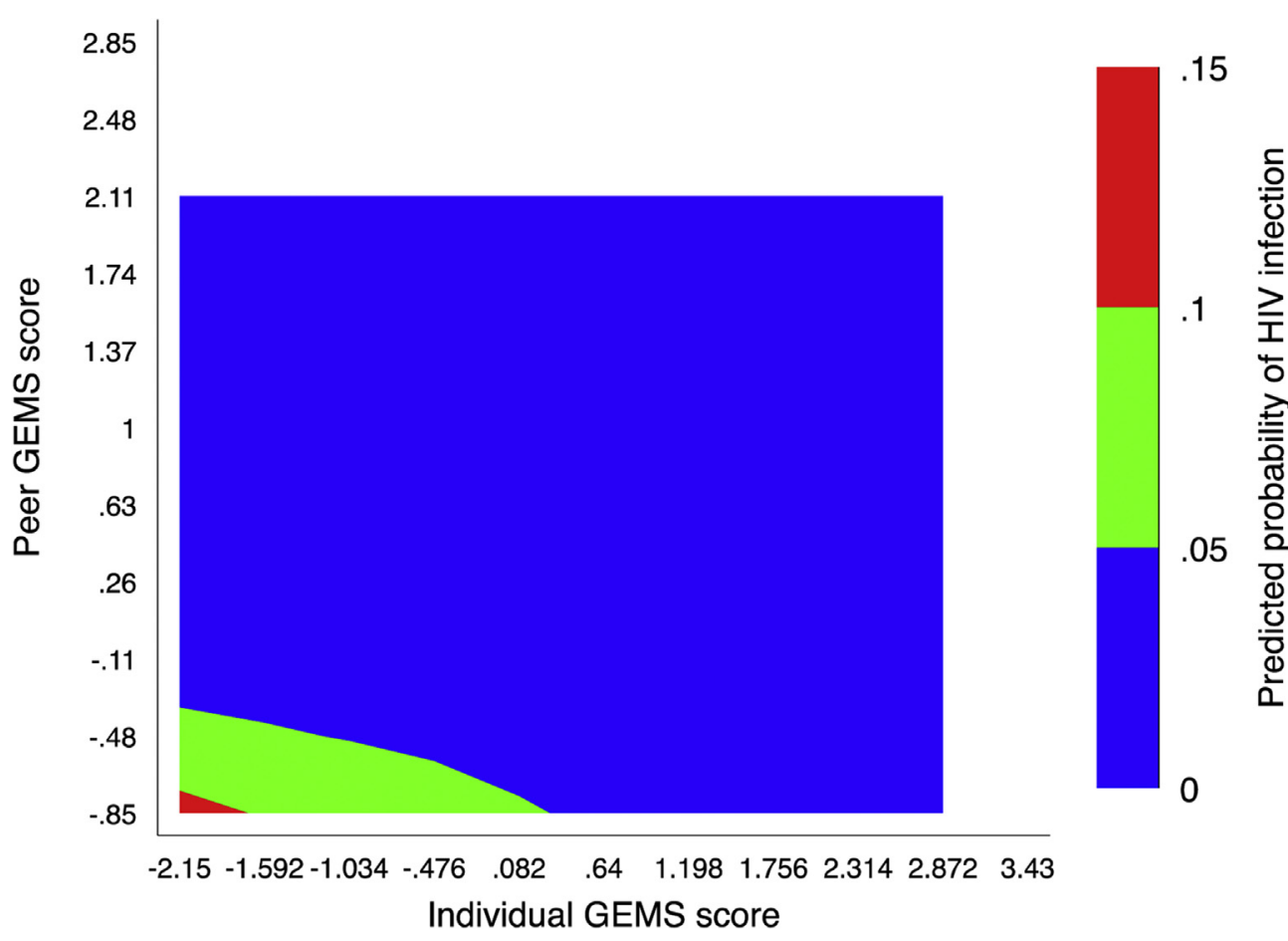

Figure 3. Contour plot showing interaction effect of individual and peer GEMS score on HIV incidence. Units for the axes on the contour plot are logits, estimated from the item response models. Predicted probabilities are in reference to a five-year period of observation.

analysis and lagging the GEMS exposure to the previous study visit. Despite these strengths, our analysis cannot fully benefit from the randomization design of HPTN 068 and therefore may be vulnerable to unmeasured confounding. We purposely selected confounders based on related studies using the GEMS instrument in South Africa and variables known to be influential in the HPTN 068 context. However, the possibility for unmeasured confounding, influencing multilevel GEMS scores and individual risk for HIV and HSV-2 acquisition, remains.

Finally, the finding that individual GEMS scores were not directly associated with risk of HIV and HSV-2 acquisition could be due to insufficient statistical power to detect this association. Neither RCT were designed to investigate the association between individual-level GEMS scores and HIV or HSV-2 acquisition among AGYW. Given the relatively small magnitude of association with peer GEMS scores, it is possible that a larger sample size of AGYW is necessary to detect a small, but significant, association between individually held gender norms and HIV/HSV-2 acquisition.

Gender-equitable norms and roles are modifiable risk factors that can potentially reduce HIV and HSV-2 acquisition among AGYW [28]. Our study underscores not just the importance of the social environment for young people but specifically the importance of the broad peer environment for modifying risk in AGYW. Interventions within the peer environment that increase the level of endorsement of gender-equitable norms could have a multitude of beneficial effects for young women in South Africa, notably a significant reduction in HIV and HSV-2 acquisition.

\section{Funding Sources}

P.W. received supported from the National Institute of Mental Health (NIMH) of the U.S. Public Health Service (T32 MH19105).
This research was supported by the U.S. National Institute of Mental Health (R01MH110186; Pettifor). Data collection for HPTN 068 was provided by the National Institute of Allergy and Infectious Diseases (NIAID), the National Institute of Mental Health, and the National Institute on Drug Abuse (NIDA) of the National Institutes of Health (NIH; award numbers UM1AI068619 [HPTN Leadership and Operations Center], UM1AI068617 [HPTN Statistical and Data Management Center], and UM1AI068613 [HPTN Laboratory Center]). Community survey data were supported by NIMH (R01MH087118; Pettifor and R01MH103198; Lippman/Pettifor). The Agincourt HDSS and census data collection is supported by South African Medical Research Council and University of the Witwatersrand, as well as the Wellcome Trust, UK (grants 058893/Z/99/A; 069683/Z/02/Z; 085477/Z/08/Z; 085477/B/08/Z).

\section{References}

[1] Joint United Nations Programme on HIV/AIDS (UNAIDS): South Africa. Available at: http://www.unaids.org/en/regionscountries/countries/southafrica/. Accessed May 3, 2018.

[2] Joint United Nations Programme on HIV/AIDS (UNAIDS): Women and girls and HIV. Geneva, Switzerland: UNAIDS; 2018. Available at: http://www. unaids.org/sites/default/files/media_asset/women_girls_hiv_en.pdf. Accessed May 3, 2018.

[3] Zuma K, Shisana O, Rehle TM, et al. New insights into HIV epidemic in South Africa: Key findings from the National HIV Prevalence, Incidence and Behaviour Survey , 2012. Afr J AIDS Res 2016;15:67-75.

[4] Patton GC, Sawyer SM, Santelli JS, et al. Our future: A Lancet commission on adolescent health and wellbeing. Lancet 2016;387:2423-78.

[5] Wood K, Maforah F, Jewkes R. "He forced me to love him" Putting violence on adolescent sexual health agendas. Soc Sci Med 1998;47:233-42.

[6] Dunkle KL, Jewkes RK, Brown HC, et al. Gender-based violence, relationship power, and risk of HIV infection in women attending antenatal clinics in South Africa. Lancet 2004;363:1415-21.

[7] Kalichman SC, Simbayi LC, Cain D, et al. Sexual assault, sexual risks and gender attitudes in a community sample of South African men. AIDS Care 2007;19:20-7. 
[8] Jewkes R, Morrell R. Sexuality and the limits of agency among South African teenage women: Theorising femininities and their connections to HIV risk practices. Soc Sci Med 2012;74:1729-37.

9] Jewkes R, Morrell R. Gender and sexuality: Emerging perspectives from the heterosexual epidemic in South Africa and implications for HIV risk and prevention. J Int AIDS Soc 2010;13:1-11.

[10] Jewkes R, Levin JB, Penn-Kekana LA. Gender inequalities, intimate partne violence and HIV preventive practices: Findings of a South African crosssectional study. Soc Sci Med 2003;56:125-34.

[11] Gottert A, Barrington C, Pettifor A, et al. Measuring men's gender norm and gender role conflict/stress in a high HIV-prevalence South African setting. AIDS Behav 2016;20:1785-95.

[12] Pettifor A, Measham D, Rees H, Padian N. Sexual power and HIV risk, South Africa. Emerg Infect Dis 2004;10:1996-2004.

[13] Kahn K, Collinson MA, Gomez-Olive FX, et al. Profile: Agincourt health and socio-demographic surveillance system. Int J Epidemiol 2018;41: 988-1001.

[14] Pettifor A, Macphail C, Hughes JP, et al. The eff ect of a conditional cash transfer on HIV incidence in young women in rural South Africa ( HPTN 068 ): a phase 3 , randomised controlled trial. Lancet Glob Health 2016;4: e978-88.

[15] Lippman SA, Neilands TB, Macphail C, et al. Community mobilization for HIV testing uptake: Results from a community randomized trial of a theory-based intervention in rural South Africa. J Acquir Immune Defic Syndr 2017;74(Suppl 1):44-51.

[16] Pettifor A, Lippman SA, Gottert A, et al. Community Mobilization to modify harmful gender norms and reduce HIV risk: Results from community cluster randomized trial in South Africa. J Int AIDS Soc 2018 21:e25134.
[17] Pulerwitz J, Barker G. Measuring attitudes toward gender norms among young men in Brazil. Men Masc 2008;10:322-38.

[18] Wilson M. In: Riegert D, ed. Constructing Measures: An Item Response Modeling Approach. Mahwah, NJ: Lawrence Erlbaum Associates, Inc: 2005.

[19] Embretson SE, Yang X. Item response theory. Handb Complement Methods Educ Res 2006;14:385-409.

[20] R Core Team. R: A language and environment for statistical computing 2014. Available at: http://www.r-project.org. Accessed May 30, 2017.

[21] Robitzsch A, Kiefer T, Wu M. Test analysis Modules ('TAM'). Available at: http://www.edmeasurementsurveys.com/TAM/Tutorials/. Accessed May 30, 2017.

[22] Ludlow L, Haley S. Rasch model logits: Interpretation, use, and transformation. Educ Psychol Meas 1995;55:967-75.

[23] Pettifor A, Lippman SA, Selin AM, et al. A cluster randomized-controlled trial of a community mobilization intervention to change gender norms and reduce HIV risk in rural South Africa: Study design and intervention. BMC Public Health 2015;15:1-7.

[24] StataCorp.. Stata statistical software: Release 15. College Station, TX: StataCorp LP; 2017.

[25] Szklo M, Nieto FJ. Epidemiology: Beyond the basics. 2nd ed. Sudbury, Massachusetts: Jones and Bartlett Publishers, LLC; 2007.

[26] Gottert A, Barrington C, Luz H, et al. Gender norms, gender role conflict stress and HIV risk behaviors among men in Mpumalanga, South Africa. AIDS Behav 2017;4.

[27] Krieger N. Epidemiology and the web of causation: Has anyone seen the spider? Soc Sci Med 1994;39:887-903.

[28] Dworkin S, Treves-Kagan S, Lippman S. Gender-transformative interventions to reduce HIV risks and violence with heterosexually-active men: A review of the global evidence. AIDS Behav 2013;17:2845-63. 